\title{
A Flare of Systemic Lupus Erythematosus Disease After COVID-19 Infection: A Case of Lupus Cerebritis
}

\author{
Muhammad Zain Khalid $^{1}$, Sylvette Rogers ${ }^{2}$, Ayesha Fatima ${ }^{3}$, Manal Dawe ${ }^{4}$, Romil Singh 5 \\ 1. Internal Medicine, Liaquat National Hospital and Medical College, Karachi, PAK 2. Family Medicine, Caribbean \\ Medical University, Des Plaines, USA 3. Gynaecology \& Obstetrics, Fauji Foundation Hospital, Rawalpindi, PAK 4. \\ Internal Medicine, Capital Medical University, Beijing, CHN 5. Critical Care, Mayo Clinic, Rochester, USA
}

Corresponding author: Romil Singh, singh.romil20@gmail.com
Review began 06/09/2021 Review ended 06/20/2021 Published 07/02/2021

\section{○ Copyright 2021}

Khalid et al. This is an open access article distributed under the terms of the Creative Commons Attribution License CC-BY 4.0., which permits unrestricted use, distribution, and reproduction in any medium, provided the original author and source are credited.

\section{Abstract}

The association between coronavirus disease 19 (COVID-19) and autoimmune disease has been mounting, and literature on COVID-19-induced flare-up of systemic lupus erythematosus (SLE) disease is lacking. We describe a case of lupus cerebritis triggered by COVID-19 in a young female with SLE, who presented with fluctuated mentation, psychomotor retardation, slow speech, and intermittent choreiform movement in the upper part of the body. She had a history of COVID-19 infection three weeks back. Her serum immunoglobulin $\mathrm{G}$ antibodies were positive against COVID-19. On examination, she had psychomotor agitation, intermittent choreiform movements of upper limbs, and poor speech. Brain magnetic resonance imaging revealed hyperintense signals in the white matter of both hemispheres, suggestive of lupus cerebritis secondary to COVID-19 infection and lack of any other identifiable risk factor. Management included methylprednisolone, prednisone, and olanzapine. The patient was also placed on monthly intravenous cyclophosphamide, and her condition started improving gradually.

Categories: Internal Medicine, Neurology

Keywords: covid-19, sars-cov-2, sle, lupus cerebritis, systemic lupus erythematosus disease

\section{Introduction}

Severe acute respiratory syndrome coronavirus 2 (SARS-CoV-2) is the causative agent of coronavirus disease 19 (COVID-19). COVID-19 has a broad spectrum of clinical manifestations involving different systems of the body [1]. COVID-19 generally presents with signs and symptoms of the respiratory system, including flu-like illness complicated by acute respiratory distress syndrome (ARDS) and lung failure [2]. Other manifestations and complications include severe metabolic syndrome, acute kidney injury, neurological syndromes, cardiovascular and thromboembolic events such as encephalopathy, seizures, and stroke [3-7]. A possible association between COVID-19 and autoimmune disease has also been reported in many case reports [8]. Systemic lupus erythematosus (SLE) disease has been reported in patients with COVID-19 [9]. Herein, we describe a rare case of lupus cerebritis triggered by SARS-CoV-2 in a young female diagnosed with SLE.

\section{Case Presentation}

A 29-year-old female with a past medical history of SLE was brought to the emergency department with fluctuated mentation, fatigue, anorexia, and psychomotor retardation for the last week. She also complained of incoherent speech and intermittent choreiform movement in the upper part of the body. She was diagnosed with SLE four years back, having urticaria and erythematosus rash with itching, scaling of the palm of hands, and hyperkeratosis of the sole, for which she was taking hydroxychloroquine and prednisone. She was admitted to the hospital three weeks back due to worsening dyspnea, fever, and cough. She had tachypnea, wheezing, and a chest X-ray revealed diffuse infiltrates in both lungs. Her COVID-19 polymerase chain reaction (PCR) test was positive, and she was commenced on azithromycin and 6mg dexamethasone for five days. Her condition improved gradually, and she was discharged six days later.

On clinical examination, she looked anxious with poor speech. She had a temperature of $99^{\circ} \mathrm{F}$, respiratory rate of 23/minute, heart rate of $87 /$ minute, blood pressure of $110 / 70 \mathrm{mmHg}$, and oxygen saturation of $96 \%$. Her cardiovascular and respiratory examination was unremarkable, with normal vesicular breathing and heart sounds. Neurological examination revealed psychomotor agitation, intermittent choreiform movements of upper limbs, and poor speech. She had no signs of meningeal irritation, muscle strength loss, seizure episodes, and any history of trauma and illicit drug use. Her repeat COVID-19 PCR test was negative; however, serum immunoglobulin G (IgG) antibodies were positive against COVID-19.

Her initial blood investigations revealed thrombocytopenia and mild elevation of creatinine (Table 1). Infectious workup was negative for any organism. The urine screen was negative for any illicit drug use, and the result of her repeat autoimmune screening is shown in Table 2. Her brain magnetic resonance imaging (MRI) revealed hyperintense signals in the bilateral parietal and temporal lobes, suggestive of lupus 


\section{Cureus}

cerebritis (Figure 1). She was diagnosed with lupus cerebritis, an exacerbation of SLE due to COVID-19 infection. CSF analysis was not performed because she refused lumbar puncture.

\begin{tabular}{|c|c|c|}
\hline Parameter & Lab value & Reference range \\
\hline White blood cell count & 10,600 & $4,000-11,000 \mathrm{~mm}^{3}$ \\
\hline Platelet count & 135,000 & $150,000-350,000 \mathrm{~mm}^{3}$ \\
\hline Red blood cell count & 3.9 & 04-06 million cells $/ \mathrm{mm}^{3}$ \\
\hline Hemoglobin & 11.9 & $11.5-17.5 \mathrm{mg} / \mathrm{dL}$ \\
\hline Erythrocyte sedimentation rate & 23 & $0-20 \mathrm{~mm} / \mathrm{hour}$ \\
\hline C-reactive protein & 11 & $<10 \mathrm{mg} / \mathrm{L}$ \\
\hline Creatinine & 1.4 & $0.9-1.1 \mathrm{mg} / \mathrm{dL}$ \\
\hline Blood urea nitrogen & 23 & $18-45 \mathrm{mg} / \mathrm{dL}$ \\
\hline Aspartate aminotransferase & 33 & 08-35 IU/L \\
\hline Alanine aminotransferase & 29 & $10-40$ IU/L \\
\hline Glycosylated hemoglobin & $6-1$ & $5.7 \%-6.4 \%$ \\
\hline
\end{tabular}

\section{TABLE 1: Initial blood investigations}

\begin{tabular}{|c|c|c|}
\hline Parameter & Lab value & Reference range \\
\hline Complement $\mathrm{C} 3$ protein & 88 & $90-10 \mathrm{mg} / \mathrm{dL}$ \\
\hline Complement C4 protein & 10 & $10-40 \mathrm{mg} / \mathrm{dL}$ \\
\hline Anti-dsDNA Ab & 55 & $<35 \mathrm{IU} / \mathrm{mL}$ \\
\hline Anti-CCP Ab & Negative & Negative \\
\hline Anticardiolipin Ab & Negative & Negative \\
\hline Lupus anticoagulant & Negative & Negative \\
\hline anti- $\beta 2 G P 1 A b$ & Negative & Negative \\
\hline \multicolumn{3}{|c|}{ TABLE 2: Autoimmune screening for SLE } \\
\hline \multicolumn{3}{|c|}{$\begin{array}{l}\text { Anti-dsDNA: anti-double-stranded deoxyribonucleic acid, Ab: antibody, Anti-CCP: anti-cyclic citrullinated peptides, Anti- } \beta 2 \text { GP1: anti-beta-2 } \\
\text { glycoprotein } 1\end{array}$} \\
\hline
\end{tabular}




\section{Cureus}

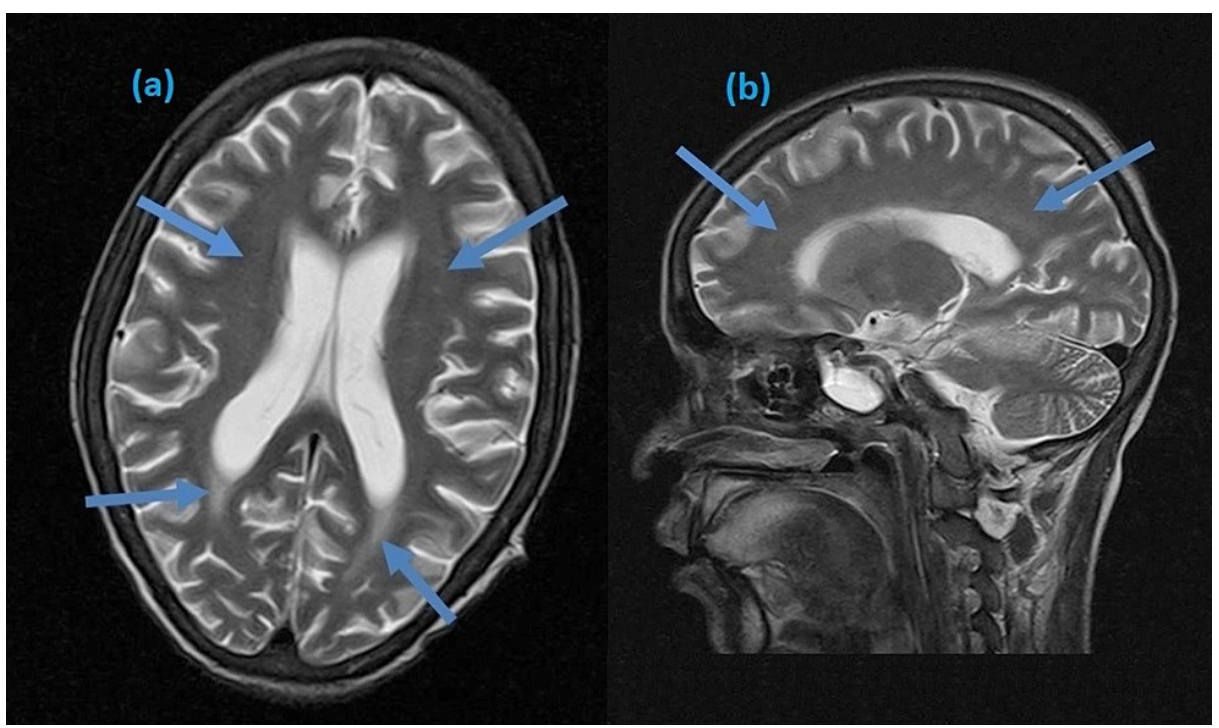

FIGURE 1: Brain MRI showing hyperintense signals in the temporal and parietal regions in axial (a) and sagittal (b) planes

Her initial management included 1g methylprednisolone for three consecutive days, 30mg of prednisone daily, and $5 \mathrm{mg}$ of olanzapine daily for two weeks. The patient was also placed on monthly $1 \mathrm{~g}$ intravenous cyclophosphamide. Her condition started improving gradually, and she was stable on day six of hospitalization. She was discharged on maintenance oral steroid therapy with follow-up.

\section{Discussion}

SLE is a multisystem disease and has complex clinical manifestations involving several organs in the body such as the heart (lupus carditis), kidney (lupus nephritis), brain (lupus cerebritis), skin, eyes, joints, and muscles [10]. SLE is an autoimmune disease characterized by the production of pathognomonic autoantibodies. Many viruses have been implicated in the etiology of SLE [11]. Epstein-Barr virus, cytomegalovirus, retroviruses, and parvovirus B19 are the possible triggers of SLE [10,11]. SLE triggered by COVID-19 has also been reported in many cases [12]. SLE patients are at increased risk of infections due to prolonged use of immunosuppressive drugs and autoimmune syndromes. Infections can exacerbate SLE activity and are reported to be a significant cause of death among SLE patients (37.7\%) [13]. SLE patients are susceptible to COVID-19 infection and can worsen the activity of SLE. Alharthy et al. reported a case of COVID-19 infection with a flare of SLE [14]. Our patient also had SLE and presented with neuropsychiatric manifestations (lupus cerebritis) triggered by COVID-19.

COVID-19 is an inflammatory disease leading to a widespread immune response throughout the body. Literature is lacking on the association between COVID-19 and SLE and the flare of SLE. Several studies have linked COVID-19 with other autoimmune diseases such as rheumatoid arthritis and multiple sclerosis. Joo et al. highlighted a link between exposure to coronavirus, parainfluenza, and metapneumovirus for six to seven weeks and increased the incidence of rheumatoid arthritis [8]. Lupus cerebritis due to COVID-19 may be due to angiotensin-converting enzyme 2 (ACE 2) receptors in the central nervous system, including basal ganglia, hippocampus, and glial cells. SARS-CoV-2 induces a severe immune response, resulting in the release of inflammatory and pro-inflammatory cytokines and chemokines, disrupting the blood-brain barrier and leading to neuropsychiatric manifestations [2]. Another mechanism may be microangiopathy arising from cytokine storm and complement activation leading to cerebral ischemia and microinfarction [12]. The cytokine storm induced by SARS-CoV-2 leads to immune-mediated damage to neuronal cells by molecular mimicry such as the production of autoantibodies like antinuclear antibodies (ANAs) [14].

The diagnosis of lupus cerebritis is based on clinical presentation, serum chemistry (complete blood count), immunological studies (ANAs and anti-dsDNA antibodies), and brain imaging using computed tomography and MRI [15]. Cerebrospinal fluid analysis is occasionally done to rule any infectious cause of neuropsychiatric manifestations. Electroencephalography has a low sensitivity and specificity for diagnosis. Management depends on the severity of the disease [15]. For mild to moderate illness, glucocorticoids in combination with azathioprine or mycophenolate are used. Intravenous cyclophosphamide can be added in severe cases [16]. Antimalarials are used as alternatives to steroids or as supplements to accelerate the steroid taper. In resistant cases, rituximab, plasmapheresis, and intravenous immunoglobulins can be used. Antipsychotics such as olanzapine can be used in case of severe psychosis [16]. 


\section{Conclusions}

Our patient was a known case of SLE. Her neuropsychiatric manifestation following COVID-19 infection, findings on brain MRI, and the improvement in her condition on starting appropriate treatment justify the flare of SLE as lupus cerebritis secondary to SARS-CoV-2. Our case describes the possible role of COVID-19 in the flare of SLE, albeit single case observations have limitations. There is a dire need for data to support this hypothesis, and more cases of autoimmune disorders are required to declare themselves following COVID-19 infection.

\section{Additional Information \\ Disclosures}

Human subjects: Consent was obtained or waived by all participants in this study. NA issued approval NA. Conflicts of interest: In compliance with the ICMJE uniform disclosure form, all authors declare the following: Payment/services info: All authors have declared that no financial support was received from any organization for the submitted work. Financial relationships: All authors have declared that they have no financial relationships at present or within the previous three years with any organizations that might have an interest in the submitted work. Other relationships: All authors have declared that there are no other relationships or activities that could appear to have influenced the submitted work.

\section{References}

1. Wu Z, McGoogan JM: Characteristics of and important lessons from the coronavirus disease 2019 (COVID19) outbreak in China: summary of a report of 72314 cases from the Chinese Center for Disease Control and Prevention. JAMA. 2020, 323:1239-42. 10.1001/jama.2020.2648

2. Zavascki AP, Falci DR: Clinical characteristics of Covid-19 in China . N Engl J Med. 2020, 382:1859.

3. Khan I, Sarwar A, Ahmed Z: Atypical case of COVID-19 associated Kawasaki disease in an eight-year-old Pakistani boy. Cureus. 2020, 12:e10670. 10.7759/cureus.10670

4. Singh R, Kashyap R, Hutton A, Sharma M, Surani S: A review of cardiac complications in coronavirus disease 2019. Cureus. 2020, 12 :e8034. 10.7759/cureus.8034

5. Menon T, Gandhi SAQ, Tariq W, et al.: Impact of chronic kidney disease on severity and mortality in COVID19 patients: a systematic review and meta-analysis. Cureus. 2021, 13:e14279. 10.7759/cureus.14279

6. Menon T, Sharma R, Kataria S, et al.: The association of acute kidney injury with disease severity and mortality in COVID- 19: a systematic review and meta-analysis. Cureus. 2021, 13:e13894. 10.7759/cureus.13894

7. Singh R, Shiza ST, Saadat R, Dawe M, Rehman U: Association of Guillain-Barre syndrome with COVID- 19: a case report and literature review. Cureus. 2021, 13:e13828. 10.7759/cureus.13828

8. Joo YB, Lim YH, Kim KJ, Park KS, Park YJ: Respiratory viral infections and the risk of rheumatoid arthritis . Arthritis Res Ther. 2019, 21:199. 10.1186/s13075-019-1977-9

9. Gartshteyn Y, Askanase AD, Schmidt NM, et al.: COVID-19 and systemic lupus erythematosus: a case series . Lancet Rheumatol. 2020, 2:e452-4. 10.1016/S2665-9913(20)30161-2

10. Zamani B, Shayestehpour M, Esfahanian F, Akbari H: The study of factors associated with pregnancy outcomes in patients with systemic lupus erythematosus. BMC Res Notes. 2020, 13:185. 10.1186/s13104020-05039-9

11. Ramos-Casals M: Viruses and lupus: the viral hypothesis. Lupus. 2008, 17:163-5. 10.1177/0961203307086268

12. Zamani B, Moeini Taba SM, Shayestehpour M: Systemic lupus erythematosus manifestation following COVID-19: a case report. J Med Case Rep. 2021, 15:29. 10.1186/s13256-020-02582-8

13. Fei Y, Shi X, Gan F, et al.: Death causes and pathogens analysis of systemic lupus erythematosus during the past 26 years. Clin Rheumatol. 2014, 33:57-63. 10.1007/s10067-013-2383-3

14. Alharthy A, Faqihi F, Nasim N, et al.: COVID-19 in a patient with a flare of systemic lupus erythematosus: a rare case-report. Respir Med Case Rep. 2020, 31:101252. 10.1016/j.rmcr.2020.101252

15. Fanouriakis A, Tziolos N, Bertsias G, Boumpas DT: Update on the diagnosis and management of systemic lupus erythematosus. Ann Rheum Dis. 2021, 80:14-25. 10.1136/annrheumdis-2020-218272

16. Magro-Checa C, Zirkzee EJ, Huizinga TW, Steup-Beekman GM: Management of neuropsychiatric systemic lupus erythematosus: current approaches and future perspectives. Drugs. 2016, 76:459-83. 10.1007/s40265015-0534-3 